\title{
Electric and Magnetic Properties of Hydrogels Doped with $\mathrm{Cu}$ Ions
}

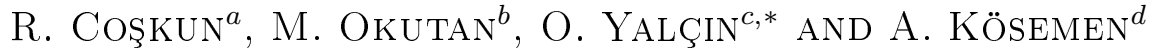 \\ ${ }^{a}$ Department of Chemistry, Bozok University, 66500, Yozgat, Turkey \\ ${ }^{b}$ Department of Physics, Yildiz Technical University, 34210 Istanbul, Turkey \\ ${ }^{c}$ Department of Physics, Niğde University, 51240, Niğde, Turkey \\ ${ }^{d}$ Department of Physics, Muş Alparslan University, 49100, Muş, Turkey
}

(Received December 20, 2011; in final form June 25, 2012)

\begin{abstract}
The electric and magnetic properties of $\mathrm{Cu}$ doped and pure/undoped hydrogels were investigated by impedance spectroscopy and electron spin resonance techniques, respectively. The capacitance and dissipation factor $(\tan \delta$ ) were measured in the frequency range of $100 \mathrm{~Hz}-10 \mathrm{MHz}$ at room temperature. The Cole-Cole plots for the $\mathrm{Cu}$ doped dry and pure dry hydrogels have been used to describe the characteristic change of electrical properties in mentioned temperature interval. The comparison between $\mathrm{Cu}$ ions doped and pure/undoped samples is done using the impedance spectroscopy and the electron spin resonance techniques. Electron spin resonance signal for pure, $\mathrm{Cu}$ doped wet and $\mathrm{Cu}$ doped dry samples are not any power absorption signal, near the symmetric with respect to the resonance field values according to the dry sample and asymmetric for resonance field/base line, respectively.
\end{abstract}

PACS: 52.25.Mq, 82.35.-x, 42.70.Jk, 64.60.-i, 76.30.-v

\section{Introduction}

Hydrogels have received increasing interest in the preparing [1-4] and investigation of the drug delivery applications in the last years. Hydrogels have potential applications in broad range of topical areas, biology, biomedical, photo-electronics, life sciences, biosensors, some ecological problems [5-9], and tissue engineering [10-12]. Hydrogels have been prepared using a variety of techniques [5, 13-15]. Some metal-containing polymers can be considered a new generation of materials with a potentially wide application in fields such as liquid crystals $[16,17]$, smart materials, superconducting materials and biocompatible polymers [18-23]. However, dielectric and magnetic properties of pure, Cu-ions-doped-wet and Cu-ions-doped-dry hydrogels have not been studied. There is no detailed information in literature about the electric and magnetic properties of this type hydrogels. The impedance spectroscopy (IS) and electron spin resonance (ESR) have become very popular and powerful techniques for studying the dielectric and magnetic properties for this type hydrogels, respectively.

In the focus on this work, we have studied electric and magnetic properties of pure, $\mathrm{Cu}$-ions-doped-wet and $\mathrm{Cu}$ -

\footnotetext{
* corresponding author; e-mail: o.yalcin@nigde.edu.tr, orhanyalcin@gmail.com
}

-ions-doped-dry hydrogels by using the impedance spectroscopy (or dielectric spectroscopy) (IS\&DC) and ESR techniques, respectively. The frequency evolution of the real $\left(\varepsilon^{\prime}\right)$ and imaginary part $\left(\varepsilon^{\prime \prime}\right)$ of the dielectric constant for pure, $\mathrm{Cu}$-ions-doped-wet and $\mathrm{Cu}$-ions-doped-dry hydrogels were studied in the frequency range $100 \mathrm{~Hz}$ to $10 \mathrm{MHz}$. The change of $\varepsilon^{\prime \prime}$ with frequency gives information on the dielectric relaxation frequency and loss of the energy level about the samples. Doping on hydrogels was shown drastic change on the dielectric constant. The main aims of this work are to investigate electric and magnetic properties of pure, $\mathrm{Cu}$-ions-doped-wet and Cu-ions-doped-dry hydrogels.

\section{Experimental details}

\subsection{Sample preparation}

\subsubsection{Synthesis of hydrogels}

The poly (2-acrylamido-2-methylpropane sulphonic acid-co-itaconic acid, or poly (AMPS-co-IA)), hydrogels were synthesized by co-polymerization of AMPS and IA in aqueous medium, using $\mathrm{N}, \mathrm{N}^{\prime}$-methylenebisacrylamide (MBAAm) as cross linker and potassium peroxodisulfate (KPS), as initiator. In brief, $9.0 \mathrm{mmol}$ of AMPS, $1.0 \mathrm{mmol}$ of IA, and $1.0 \mathrm{mmol}$ of crosslinker were dissolved in distilled water to give a total volume of $5.0 \mathrm{ml}$. After adding $0.15 \mathrm{mmol}$ of initiator, the resulting solution was placed in poly (vinylchloride) straws of $0.40 \mathrm{~cm}$ 
diameters and about $20 \mathrm{~cm}$ long. The poly (vinylchloride) straws were sealed and immersed in a thermostated water bath at $50^{\circ} \mathrm{C}$. Polymerization was conducted for $24 \mathrm{~h}$. After the reaction, the hydrogels were cut into specimens of approximately $10 \mathrm{~mm}$ in length and dried in an oven at $55{ }^{\circ} \mathrm{C}$ to constant weight and subjected to Soxhlet extraction with water to remove the uncross-linked polymer from copolymers. The extracted gels were dried again in oven at $55^{\circ} \mathrm{C}$ to constant weight.

\subsubsection{Metal loading study}

Dried samples $(0.1 \mathrm{~g}$ each) of hydrogels were added in $100 \mathrm{ml}$ Erlenmeyer including volumes of $30 \mathrm{~cm}^{3}$ of the $\mathrm{Cu}^{2+}$ ions solution (25 ppm) adjusted to desired $\mathrm{pH}$. The mixture was stirred through the experiment at $25^{\circ} \mathrm{C}$ for $24 \mathrm{~h}$. The $\mathrm{Cu}^{2+}$ ions loaded hydrogels were taken at the end of loading. Thereafter, the hydrogels were washed with deionized water and dried.

\subsection{Dielectric measurements}

Alternative current (AC) electric properties were analyzed from the capacitance and the dissipation factor values. HP 4194A Impedance analyzer was utilized during these measurements. The complex dielectric response was considered between $100 \mathrm{~Hz}$ and $10 \mathrm{MHz}$. In this study, the overall errors for the real and imaginary parts of the complex dielectric permittivity in the low and high-frequency region were less than $2.5 \%$. The root mean square (rms) amplitude of the device is $\approx 495 \mathrm{mV}$.

\subsection{ESR measurements}

ESR spectra of pure, Cu-ions-doped-wet and Cu-ions-doped-dry hydrogels samples were taken by high sensitive conventional X-band $(9.5 \mathrm{GHz})$ commercial Bruker EMX-type spectrometer at room temperature (RT). The field derivative ESR spectra of these samples are given in Fig. 1.

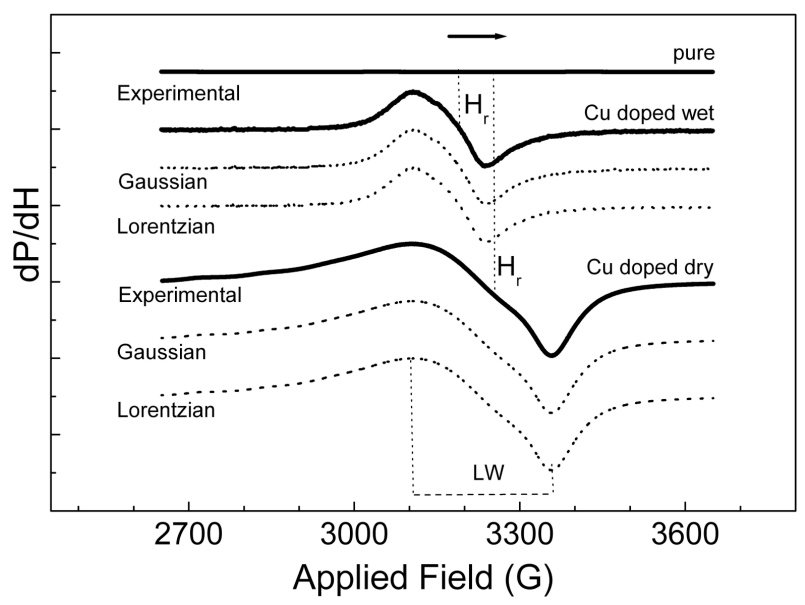

Fig. 1. ESR spectra of pure, Cu-ions-doped-wet and $\mathrm{Cu}$-ions-doped-dry samples and their theoretical Gaussian and Lorentzian simulations.

\section{Results and discussions}

\subsection{Electrical properties}

The complex dielectric permittivity $\varepsilon$ is defined as $\varepsilon=\varepsilon^{\prime}+\mathrm{i} \varepsilon^{\prime \prime}$. Here, $\varepsilon^{\prime}$ and $\varepsilon^{\prime \prime}$ are accordingly referred to as the real and imaginary parts of the complex $\varepsilon$, and are represented by $\operatorname{Re}(\varepsilon)$ and $\operatorname{Im}(\varepsilon)$, respectively. The complex conjugates of $\varepsilon$ is denoted as $\varepsilon^{*}, \varepsilon^{*}=\varepsilon^{\prime}-\mathrm{i} \varepsilon^{\prime \prime}$. The product of a complex number and its conjugate are real numbers $\varepsilon \varepsilon *=\left(\varepsilon^{\prime}\right)^{2}+\left(\varepsilon^{\prime \prime}\right)^{2}$. Therefore the complex conjugates of dielectric respectively can be written as [24-26]:

where

$$
\varepsilon^{*}(\omega)=\varepsilon^{\prime}(\omega)-\mathrm{i} \varepsilon^{\prime \prime}(\omega)=\varepsilon_{\infty}+\frac{\varepsilon_{\mathrm{S}}-\varepsilon_{\infty}}{1+(\mathrm{i} \omega \tau)^{1-\alpha}},
$$

$$
\begin{aligned}
& \varepsilon^{\prime}(\omega)=\varepsilon_{\mathrm{S}}+\left(\varepsilon_{\infty}-\varepsilon_{\mathrm{S}}\right) \\
& \quad \times \frac{1+(\omega \tau)^{1-\alpha} \sin (\pi \alpha / 2)}{1+2 \sin (\pi \alpha / 2)(\omega \tau)^{1-\alpha}+(\omega \tau)^{2(1-\alpha)}}
\end{aligned}
$$

and

$$
\begin{aligned}
& \varepsilon^{\prime \prime}(\omega)=\left(\varepsilon_{\infty}-\varepsilon_{\mathrm{S}}\right) \\
& \quad \times \frac{(\omega \tau)^{1-\alpha} \sin (\pi \alpha / 2)}{1+2 \sin (\pi \alpha / 2)(\omega \tau)^{1-\alpha}+(\omega \tau)^{2(1-\alpha)}} .
\end{aligned}
$$

In this expression, $\varepsilon_{\infty}, \varepsilon_{\mathrm{S}}$ are high and static (low) frequency dielectric constants, respectively. The parameter, $\alpha$, changes from zero to one $(0<\alpha \leq 1)$ and this depends on temperature. If $\alpha=0$, it corresponds to standard Debye type relation. The frequency evolution of the real $\left(\varepsilon^{\prime}\right)$ and imaginary part $\left(\varepsilon^{\prime \prime}\right)$ of the dielectric constant for hydrogels were studied according to the frequency range $100 \mathrm{~Hz}$ to $10 \mathrm{MHz}$. But the parameter, $\alpha$, has values different from zero for all temperatures. Therefore, the Debye type relation may not been seen in this study. Here the frequency used is the angular frequency; the real part of complex dielectric constant $\varepsilon^{\prime}$ of a material is the normalized permittivity with respect to the permittivity of vacuum. It is a measure of the amount of polarization in a material and calculated from the following equation:

$$
\varepsilon^{\prime}=\frac{C_{\mathrm{p}} d}{\varepsilon_{0} A},
$$

where $C_{\mathrm{p}}$ is the parallel capacitance in the electrode, $d$ is the inter electrodes distance, $\varepsilon_{0}$ is the permittivity of free space, $A$ is the sample area.

The frequency evolution of the real part $\left(\varepsilon^{\prime}\right)$ of the dielectric constant in the experimental results for undoped and $\mathrm{Cu}$-doped hydrogels are given in Fig. 2. Starting from the $100 \mathrm{~Hz}$, the $\varepsilon^{\prime}$ values for pure and $\mathrm{Cu}$-doped dry hydrogels are slowly decreasing while the frequency is increasing. A slow decrease for $\mathrm{Cu}$ doped hydrogels was observed smaller than for undoped hydrogels according to the frequency. On the other hand, the real part, $\varepsilon^{\prime}$, of the dielectric constant has decreased with $\mathrm{Cu}$-doping. At low frequency, $\varepsilon^{\prime}$ value corresponds to 231100 and 67722 for undoped and $\mathrm{Cu}$-doped hydrogels, respectively. Level of the $\varepsilon^{\prime}$ value decreased by doping with $\mathrm{Cu}$ ions. From this result, $\mathrm{Cu}$ ions doping provided extra value 


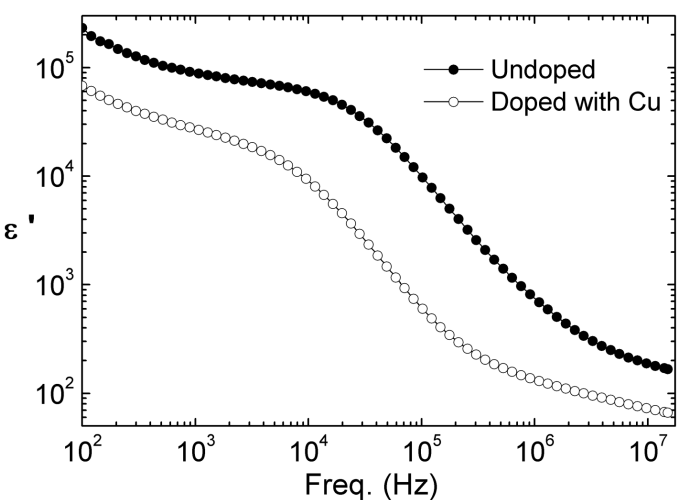

Fig. 2. The frequency evolution of the real part of the complex dielectric constant $\mathrm{Cu}$ ions doped and pure/ undoped hydrogels $\left(\varepsilon^{\prime}-f\right)$.

for the conductivity. The real part of $\varepsilon_{\mathrm{S}}$ (minimum) and $\varepsilon_{\infty}$ (maximum) dielectric values of complex dielectric, $\varepsilon$, have been shown in Table.

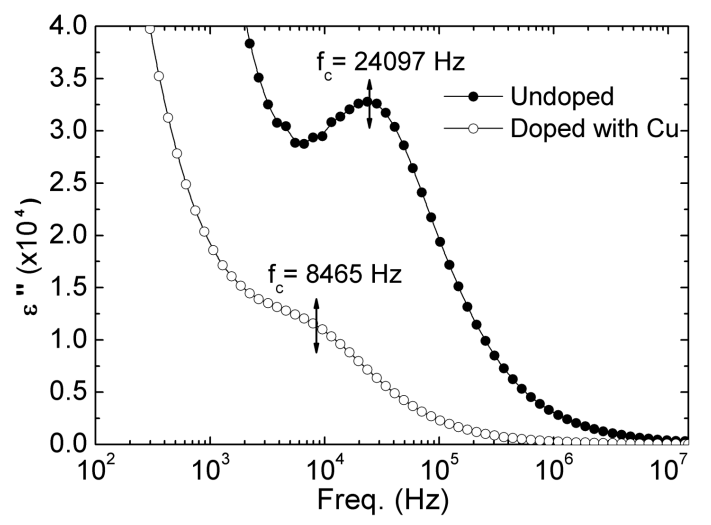

Fig. 3. The frequency evolution of the imaginary part of complex dielectric constant for $\mathrm{Cu}$ ions doped and pure/undoped hydrogels $\left(\varepsilon^{\prime \prime}-f\right)$.

$\varepsilon_{\mathrm{S}}, \varepsilon_{\infty}, \varepsilon_{\max }$, and $\tau(s)$ values.

TABLE

\begin{tabular}{c|c|c|c|c}
\hline \hline Samples & $\varepsilon_{\mathrm{S}}$ & $\varepsilon_{\infty}$ & $\varepsilon_{\max }$ & $\tau(s)$ \\
\hline undoped & 231100 & 167.14 & 32806.5 & $6.60476 \times 10^{-6}$ \\
Cu-doped & 67722 & 66.232 & 12262.9 & $1.88015 \times 10^{-5}$
\end{tabular}

Figure 3 shows the evolution of the imaginary part $\left(\varepsilon^{\prime \prime}\right)$ of the complex dielectric constant in the experimental results for pure/undoped and $\mathrm{Cu}$ ions doped dry hydrogels. It can be seen from Fig. 3 that an exponential increase in imaginary part $\left(\varepsilon^{\prime \prime}\right)$ of the complex dielectric constant can be identified for pure/undoped and $\mathrm{Cu}$ ions doped hydrogels with decreasing frequency. Near this behavior, another increase of imaginary part $\left(\varepsilon^{\prime \prime}\right)$ of the complex dielectric permittivity has been observed around the $8465 \mathrm{~Hz}$ and $24097 \mathrm{~Hz}$ for $\mathrm{Cu}$ ions doped and undoped samples, respectively. These maximum in the $8465 \mathrm{~Hz}$ and $24097 \mathrm{~Hz}$ originated from level of the energy loss for each hydrogels. The peak frequency shifts to higher frequencies with an increase frequency for undoped samples. The imaginary part of the complex dielectric constant peak and its shifts with undoping suggest a dielectric relaxation process. The dielectric relaxation frequency decrease and maximum peak values disappeared for $\mathrm{Cu}$ ions doped hydrogels. In additional, critical frequency values slip to low frequency region with $\mathrm{Cu}$-doped hydrogels. This behavior is seen from Fig. 3 in detail for $24097 \mathrm{~Hz}$ and $8465 \mathrm{~Hz}$ for pure/undoped and Cu-doped hydrogels, respectively. Relaxation times $(\tau)$ were measured by using the $\varepsilon^{\prime \prime}-f$ figures (Fig. 3).

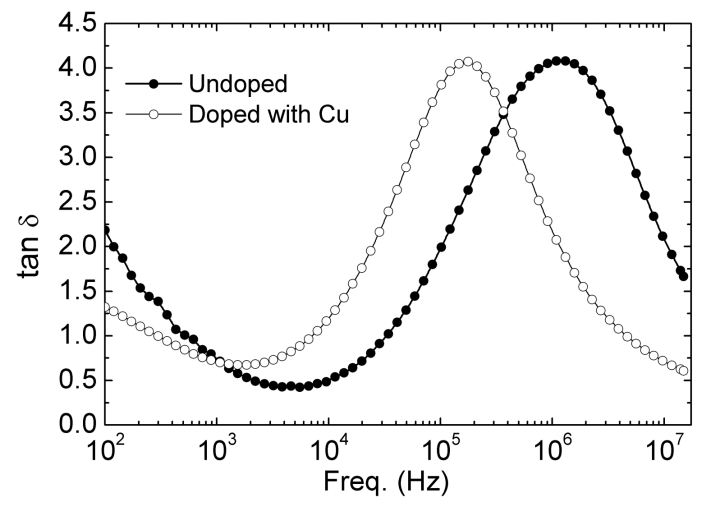

Fig. 4. Dependence of the dissipation factor vs. frequency $(\tan \delta-f)$ plots.

Moreover, the energy or dielectric loss, $\varepsilon^{\prime \prime}$, was calculated from Eq. (3)

$$
\tan \delta=\frac{\varepsilon^{\prime \prime}}{\varepsilon^{\prime}}
$$

where $\delta=90^{\circ}-\varphi$ and $\varphi$ is the phase angle. Tangent $\operatorname{loss} \tan \delta$ is also typically considered as a characteristic dielectric quantity. This behavior is seen in Fig. 3 in detail by using the $\tan \delta-f$. Energy loss factor decreases with $\mathrm{Cu}$ ions doped hydrogels from maximum peaks in Fig. 4. Maximum values of the dissipation factor for pure/undoped and $\mathrm{Cu}$-doped hydrogels correspond to $1155962 \mathrm{~Hz}$ and $170329 \mathrm{~Hz}$, respectively. The maximum values of $\tan \delta$ values of $\mathrm{Cu}$ doped sample smaller than the pure/undoped sample. In additional, for the narrowing of maximum peaks in $\mathrm{Cu}$ doped hydrogels, condition changes from the molecular vibration to rotational motion. The contributions of different resistance can be separated by complex impedance measurement. This result corresponds to a parallel circuit with the component resistance $R$ and the component capacitance $C$. The complex dielectric permittivity $\varepsilon\left(\varepsilon=\varepsilon^{\prime}+\mathrm{i} \varepsilon^{\prime \prime}\right)$ is expressed by the Debye type $(\alpha=0)$ equation

$$
\varepsilon=\varepsilon_{\infty}+\frac{\varepsilon_{\mathrm{S}}-\varepsilon_{\infty}}{1+\mathrm{i} \omega \tau} .
$$

Here $\mathrm{i}$ and $\omega$ are the imaginary number unit and angle frequency, respectively. The real $\varepsilon^{\prime}$ and the imaginary 
part $\varepsilon^{\prime \prime}$ of the complex dielectric permittivity are

$$
\varepsilon^{\prime}=\varepsilon_{\infty}+\frac{\varepsilon_{\mathrm{S}}-\varepsilon_{\infty}}{1+\mathrm{i} \omega^{2} \tau^{2}}
$$

and

$$
\varepsilon^{\prime \prime}=\frac{\omega \tau\left(\varepsilon_{\mathrm{S}}-\varepsilon_{\infty}\right)}{1+\mathrm{i} \omega^{2} \tau^{2}},
$$

respectively. Thus, the relationship between $\varepsilon^{\prime}$ and $\varepsilon^{\prime \prime}$ can be expressed by

$$
\left(\varepsilon^{\prime}-\left(\varepsilon_{\infty}+\frac{\varepsilon_{\mathrm{S}}-\varepsilon_{\infty}}{2}\right)\right)^{2}+\left(\varepsilon^{\prime \prime}\right)^{2}=\left(\frac{\varepsilon_{\mathrm{S}}-\varepsilon_{\infty}}{2}\right)^{2} .
$$

This equation corresponds to circle [27]. Thus, the ColeCole plots (the imaginary part $\varepsilon^{\prime \prime}$ of the impedance is plotted as a function of the real part of the impedance $\varepsilon^{\prime}$ for $\mathrm{Cu}$ ions doped and pure/undoped hydrogels with their RC circuit are given in Fig. 5 (inset). Figure 5 shows the experimental (open circle for $\mathrm{Cu}$ ions doped and full circle for undoped samples) and theoretical (dot lines for each samples) Cole-Cole plots of the imaginary $\varepsilon^{\prime \prime}$ and real part $\varepsilon^{\prime}$ of the complex dielectric constant at room temperature. A depressed semicircle is obtained for each sample. As can be seen from Fig. 5, analysis of the Cole-Cole plots shows equivalent electrical circuit for samples. The theoretical analyses on the Cole-Cole plots were carried out and the relaxation times and dielectric parameters have been obtained according to Eq. (1) (see for details Ref. [3]). The fit parameters were given in Table.

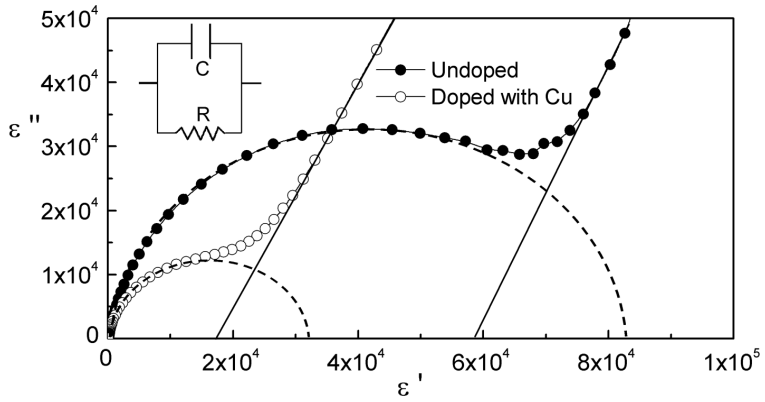

Fig. 5. Cole-Cole plots for pure/undoped and $\mathrm{Cu}$ ions doped hydrogels. An equivalent RC circuit of Cole-Cole plots is shown in the inset.

In the literature, there are standard phenomenological theories and resulting equations to analyze the Cole-Cole plots [28]. Relaxation times $(\tau)$ increase with the $\mathrm{Cu}$ ions doped. The Cole-Cole plots can be accurately presented by a RC parallel circuit model for samples. The dielectric data can be fitted to the conventional equivalent circuit. This type equivalence $\mathrm{RC}$ circuit for samples is given in inset in Fig. 5.

A specially designed computer program has been used to model for Cole-Cole plots with Eq. (1) and the ESR signal with Eqs. (5) for $\mathrm{Cu}$ doped wet and dry samples. The real part of $\varepsilon_{\mathrm{S}}$ (minimum) and $\varepsilon_{\infty}$ (maximum) dielectric values have been shown in Table.

\subsection{Magnetic properties}

The ESR data have been analyzed by using the both Lorentzian and Gaussian line shapes by analytical expressions [29]. Lorentzian and Gaussian lines are given below, respectively

$$
\frac{\mathrm{d} P_{l}}{\mathrm{~d} H}=\frac{H_{l}(0)}{1+c^{2}\left(H-H_{r}^{2}\right)^{2}}
$$

and

$$
\frac{\mathrm{d} P}{\mathrm{~d} H_{\mathrm{g}}}=H_{\mathrm{g}}(0) \exp \left(-c^{2}\left(H-H_{r}\right)^{2}\right)
$$

In these expressions; $H_{l}(0), H_{\mathrm{g}}(0), H_{r}$ and $c(c=2 / 2 \Gamma)$ denotes maximum amplitude of Lorentzian line, maximum amplitude of Gaussian line, resonance field value for each hydrogels and the inverse width factor, respectively. $2 \Gamma$ is the peak-to-peak line width.

Figure 1 shows the experimental and theoretical ESR spectra [29] for undoped (pure wet), Cu-doped-wet, and $\mathrm{Cu}$-doped-dry hydrogels. Magnetic $\mathrm{Cu}$-ions were dissolved in pure water with hydrogels. To observe an ESR signal, a single spin is not sufficient, but many of them are needed. The spin at the end of the any odd numbered $\mathrm{Cu}$-chain becomes uncoupled, giving a contribution to the ESR signal. ESR signal for pure hydrogels is not any power absorption signal. The ESR signals of $\mathrm{Cu}$-ions-doped-wet and dry hydrogels are near to symmetric with respect to the resonance field values. The resonance field and line-width of $\mathrm{Cu}$-ions-doped-dry sample are higher and broadened according to the resonance field and line-width of $\mathrm{Cu}$-ions-doped-wet sample, respectively. The resonance field values for wet and dry hydrogels are $3188 \mathrm{G}$ and $3227 \mathrm{G}$, respectively. There is also some local anomaly near the resonance field values for two Cu-ions-doped hydrogels. This anomaly can be considered as an indication to exchange interaction between the spins in the doped ions. From these results, the increase of the peak-to-peak linewidth and shift to high field region of resonance field values depend on temperature. The $g$-values of $\mathrm{Cu}$ doped wet and dry hydrogels were estimated using "ESR marker" and from the relation

$$
g=2 f(\gamma H)^{-1}
$$

with $\gamma=28 \mathrm{GHz} \mathrm{T}^{-1}$. Here, $f$ and $H$ are frequency and magnetic field, respectively. The effective $g$-values for $\mathrm{Cu}$-ions-doped wet and dry hydrogels are calculated from the scope of curve and found as $g_{\text {wet }}=2.19301$ and $g_{\text {dry }}=2.16873$. The linewidth evolution of the ESR signal for doped hydrogels is related spin glass behavior. In other words, the peak-to-peak linewidth can be determined from the spin-spin relaxation times $\left(T_{2}\right)$. The spin-spin relaxation time $\left(T_{2}\right)$ is smaller than spinorbit relaxation time $\left(T_{1}\right)$ in generally for magnetic ion doped samples. From our results, $T_{2}$ is much shorter than $T_{1}$ for $\mathrm{Cu}$-doped-dry hydrogels. The linewidth for $\mathrm{Cu}$-doped-wet hydrogels is significantly narrower compared to that for $\mathrm{Cu}$-doped dry hydrogels. In additional, this type structure corresponds to crystalline like struc- 
tures. Spin glasses behavior appears at temperature dependence. Therefore, spin glass and crystalline structure occur with increasing temperature for $\mathrm{Cu}$ doped hydrogels.

\section{Conclusion}

Values of $\varepsilon^{\prime}$ for pure and Cu-ion-doped dry hydrogels are decreasing while the frequency is increasing. The real part of the dielectric $\varepsilon^{\prime}$ constant have been decreased with $\mathrm{Cu}$-doped hydrogels. The exponential decrease in imaginary part of the $\varepsilon^{\prime \prime}$ complex dielectric constant can be identified for pure and $\mathrm{Cu}$-ion-doped dry hydrogels with increasing frequency. The dielectric relaxation frequency decrease and maximum peak values disappeared at $8465 \mathrm{~Hz}$ and $24097 \mathrm{~Hz}$ for pure and Cu-ion-doped dry hydrogels, respectively. The critical frequency values slip to low frequency region with $\mathrm{Cu}$-ion-doping. The energy loss factor $(\tan \delta)$ decrease with $\mathrm{Cu}$-ion-doped and narrowing of maximum peaks in $\mathrm{Cu}$-ion-doping hydrogels; conditions change from the molecular vibrations to rotational motion. Cu-ion-doped dry hydrogels can be considered as crystalline behavior. The spin glass state only occurs in a limited concentration range of the magnetic ions for $\mathrm{Cu}$-ion doped hydrogels. The Cole-Cole plots and electron spin resonance are good techniques in order to investigate electric and magnetic properties for hydrogels structures, respectively. Dielectric strength decreases with $\mathrm{Cu}$ ions doped hydrogels. The semicircles make their centers on a line below the real axis, which indicates the departure from the ideal Debye type behavior. From the analysis of the Cole-Cole plots, the semicircles correspond to parallel RC circuit. The relaxation time decreases with $\mathrm{Cu}$ ion doped hydrogels. The ESR signals for pure/undoped hydrogels are not any power absorption spectra.

\section{Acknowledgments}

I would like to thank Dr. Yusuf Yerli for help in the discussion of some of the results.

\section{References}

[1] J.K. Oh, D.I. Lee, J.M. Park, Prog. Polym. Sci. 34, 1261 (2009).

[2] H.A. Abd El-Rehim, Radiat. Phys. Chem. 74, 111 (2005).

[3] F. Rosso, A. Barbarisi, M. Barbarisi, O. Petillo, S. Margarucci, A. Calarco, G. Peluso, Mater. Sci. Eng. 23, 371 (2003).

[4] M. Panayiotou, R. Freitag, Polymer 46, 6777 (2005).
[5] J.M. Anderson, JM, J. Allen, G.J. Dempsey, Sensor Device 2000, US patent 6055448 .

[6] O. Wichterle, D. Lim, Nature 185, 117 (1960).

[7] H.A. Abd El-Rehim, E.A. Hegazy, H.L. Abd El-Mohdy, J. Appl. Polym. Sci. 93, 1360 (2004).

[8] E. Chow, S.Y. Lin, S.G. Johnson, P.B. Villeneuve, J.D. Joannopoulos, J.R. Wendt, G.A. Vawter, W. Zubrzycki, H. Hou, A. Alleman, Nature 407, 983 (2000).

[9] C.H. Sow, A.A. Bettiol, Y.Y.G. Lee, F.C. Cheong, C.T. Lim, F. Watt, Appl. Phys. B, Laser Opt. $\mathbf{7 8}$, 705 (2004).

[10] R. Langer, Science 293, 58 (2001).

[11] E. Soussan, S. Cassel, M. Blanzat, Angew. Chem. Int. Ed. 48, 274 (2009).

[12] B.K. Nanjawade, F.V. Manvi, A.S. Manjappa, J. Control Release 122, 119 (2007).

[13] D. Missirlis, N. Tirelli, J.A. Hubbell, Langmuir 21, 2605 (2005)

[14] V. Crescenzi, L. Cornelio, C.D. Meo, S. Nardecchia, R. Lamanna, Biomacromolecules 8, 1844 (2007).

[15] A.P. Golden, J. Tien, Lab Chip 7, 720 (2007).

[16] M. Okutan, E. Şentürk, J. Non-Cryst. Solids 354, 1526 (2008)

[17] E. Şentürk, M. Okutan, S.E. San, O. Köysal, J. Non-Cryst. Solids 354, 3525 (2008).

[18] E. Tsuchida, H. Nishide, Adv. Polym. Sci. 24, 1 (1977).

[19] F. Higashi, C.S. Cho, H. Hinoki, J. Polym. Sci. Polym. Chem. Ed. 17, 313 (1979).

[20] M.R. Buchmeiser, R. Kröll, K. Wurst, T. Schareina, R. Kempe, Macromol. Symp. 164, 87 (2001).

[21] Z.M. Michalska, K. Strzelec, J.W. Sobczak, J. Mol. Catal. A 156, 91 (2000).

[22] A. Akelah, A. Moet, Functionalised Polymers and Their Applications, Chapman and Hall, London 1990.

[23] R. Breslow, S. Belvedere, L. Gershell, D. Leung, Pure Appl. Chem. 72, 333 (2000).

[24] M.S. Cho, Y.H. Cho, H.J. Choi, M.S. Jhon, Langmuir 19, 5875 (2003).

[25] B.K.P. Scaife, Principles of Dielectrics, Clarendon Press, Oxford, U.K. 1989.

[26] Y.H. Cho, M.S. Cho, H.J. Choi, M.S. Jhon, Colloid Polym. Sci. 280, 1062 (2002).

[27] K.S. Cole, R.H. Cole, J. Chem. Phys. 9, 341 (1941).

[28] E. Şentürk, Solid State Electron. 49, 935 (2005).

[29] Jr. C.P. Poole, Electron Spin Resonance: A Comprehensive Treatise on Experimental Techniques, 2nd ed., Wiley, New York 1996. 